\title{
'Everything will eventually return to normal'
}

\author{
By Hussein Hassanali, BDJ Reader Panel, York, UK
}

W e're about three weeks in after dentistry changed overnight. These are the times where you evaluate who and/or what's important to you and how others value you. Fortunately, the practice has been very proactive from the start and taken staff welfare extremely seriously. It's been reassuring to know that your principal sees safety as a necessity as opposed to a requirement.

The initial couple of weeks when case numbers were low started to set a trend. At a time when providers and performers were frantically trying to hit contract numbers, many patients made the decisions for us. Perhaps this could be the time that highlights how performance driven targets are not conducive towards delivery of evidence-based healthcare.

As an NHS associate, the first couple of weeks were worrying from a financial perspective. Politically, things are changing so quickly that it's not possible to predict what might change from one day to the next. While NHS funding is still being fulfilled, it seems that redeployment seems more a requirement than voluntary as the only way of keeping the funding.

It's incredibly frustrating to have to manage patients with the AAA approach over the phone. We've spent so much time trying to make patients feel at ease in our presence to allow us to provide comfortable and clinically correct treatment and much of this has gone out the window. Having to convince patients that a magic pill will not solve their dental problems, yet not having a solution to provide, isn't only counterintuitive but goes against what we've been trained to do.

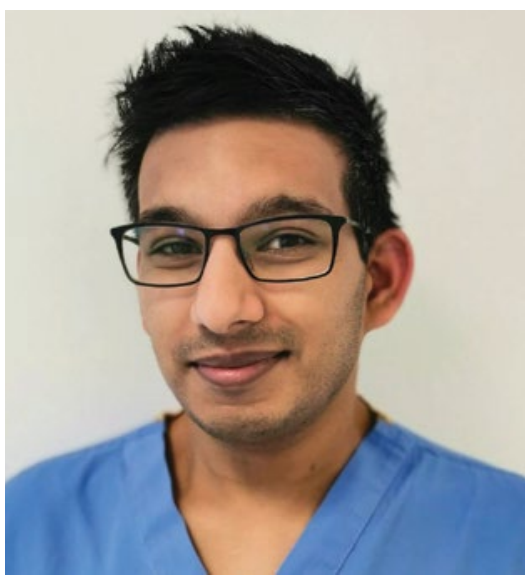

We hope that urgent dental care provisions will be ready soon. At least this will allow us to help patients in the best way we know how. Understandably, many are worried about redeployment when we hear stories of inadequate PPE. After all, we are in a profession at one of the highest risks of aerosols and contact. Inadvertently, I'd be worried of being an asymptomatic carrier and not knowing where I could have spread it to.

It's too early to tell how routine clinical dentistry will be provided in the immediate aftermath. One thing is clear: that the profession needs unified guidance and to work together for the benefits of future generations of our profession. No matter what, people will always have teeth, and our patients will need us more than ever.

Fortunately, I've got enough non-clinical work and some home projects to keep me out of trouble. I've never been a particularly materialistic person which I think has made the time I'm spending on my own easier to enjoy and finding more beauty in the world around us than the possessions we hold.

History will tell us there will be more pandemics and natural disasters in the future. History will tell us of the lessons learnt and heroes and villains. History will tell us that dentistry, healthcare, and everything else will eventually return to normal.

\section{Alumni weekend a great success}

The King's College London Dental Alumni Association hosted an incredible Alumni weekend on 6-7 March 2020 in London with bright springtime weather. Spirits were exceptionally high this year as the QS University rankings released a few days prior ranked King's as number one in the world for dentistry.

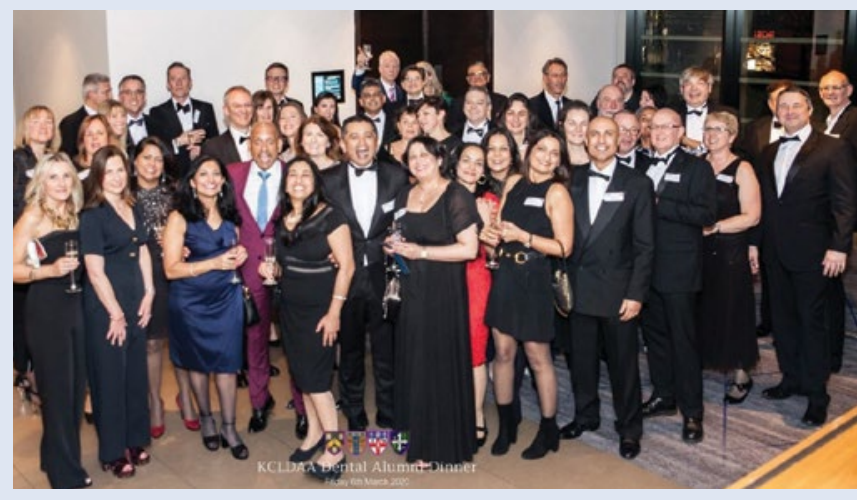

On Friday 6 March The John McLean Fellowship hosted its Annual symposium led by a panel of internationally renowned experts including Dr Subir Banerji and Professor Mike Lewis which focused on developments across the breadth of restorative dentistry, ranging from implant dentistry, minimally invasive/conservative dentistry, dental materials and oral medicine.

Concurrently, there was a Hygienist and Therapist Specialist Section Meeting held at the Gordon Museum which proved to be an invaluable addition to the day.

This was followed by the Annual Black Tie dinner which this year was held at the Hilton Tower Bridge Hotel. This provided an excellent opportunity for colleagues and friends alike to catch up over a delightful three course meal with entertainment in the form of the King's College Bhangra team, whose energetic and joyful dancing immensely impressed all the guests, even encouraging a few to jump up out of their seats and dance to the traditional Indian folk music! 
14 It was very encouraging to see the large number of recent graduates at this year's event, as the Alumni have tried to increase their reach on various social media platforms.

Other key highlights from the dinner hosted by Dr Vincent Barrett (Dental Alumni Association President) and Dr Tom Bereznicki (King's Dental Society Staff President) included a raffle organised by the Dental Alumni Association Committee with many of the prizes being kindly donated by members of staff from the Dental Institute. This was a great success, raising much needed funds towards various projects of the Alumni and the development of the facilities on Floor 31 of Guy's Tower.

The evening also included an auction which allowed lucky bidders to win a day of shadowing top dentists in their practices: Nilesh Parmar,

Tif Qureshi, Andrew Chandrapal, Andrew Dawood, Shiraz Khan and Koray Feran, with the winning bids contributing toward the student awards and prizes. This was well received and a unique, interesting feature of the night.

The lectures delivered on Saturday looked at a wide range of topics from an array of speakers including Dr Rupert Austin (Prosthodontic Clinical Tutor at King's Dental Institute), who gave a thoughtprovoking lecture on the 'Uberfication of Dentistry' - focusing on how elements of dentistry are becoming accessible through uncertified products such as direct-to-consumer clear aligners. Dr Koray Feran also gave a brilliant lecture on the importance of photography and good clinical record keeping, discussing his journey through a smile makeover case, reiterating how much thought, preparation and particularly time goes into these complex treatment plans.

These lectures were followed by another opportunity to mingle and network at the Robens Suite on Floor 29, along with tours of the undergraduate clinics following their recent refurbishment.

Overall, the weekend was deemed to be a roaring success. Not only were there ample opportunities to catch up with old friends, but also moments to network with colleagues and make new lifelong connections. The teaching was enjoyed by all, and it was great to see a large number of recent graduates as well as Alumni from years gone by.

Being a recent graduate (class of 2019), and part of the committee who helped organise the event, I was thrilled at how well the weekend went. I found the lectures most useful, and a great supplement to my everyday practice. Furthermore, it was great to catch up with colleagues I hadn't seen in a few months and talk all things dentistry. As someone fairly fresh in their career, I am excited at the prospect of being part of a tight-knit, connected Alumni and I'm already looking forward to future events.

I'd strongly encourage all Alumni to attend and become involved where they can in the future, with plans already in place to for next year's Dental Alumni Weekend on Friday 5 and Saturday 6 March 2021.

By Onkar Mudhar, Foundation Dentist, Essex

\section{BDJ $\frac{\text { RESEARCH INSIGHTS }}{\text { FOR ANOTHER VIEW }}$}

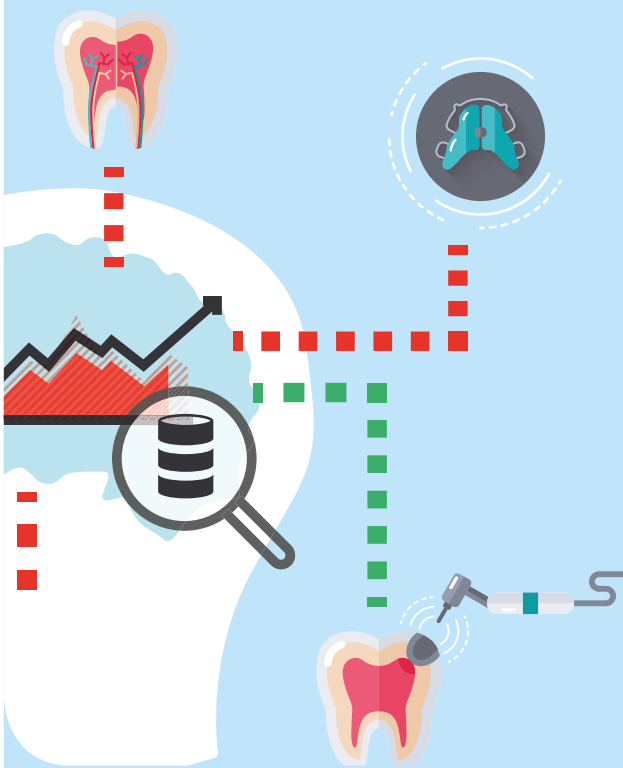

- Research insights from across the BDJ Portfolio

- Providing busy readers with valuable context including article summaries and expert commentary

Catch up here: http://bit.ly/BDJResearchlnsights

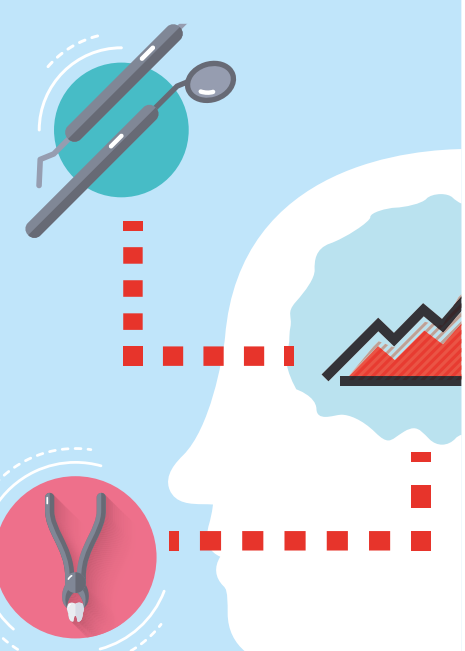

